\title{
Automated Observations of the Earthshine
}

\author{
P. R. Goode, ${ }^{1}$ S. Shoumko, ${ }^{1}$ E. Pallé, ${ }^{2}$ and P. Montañés-Rodríguez ${ }^{2}$ \\ ${ }^{1}$ Big Bear Solar Observatory, New Jersey Institute of Technology, Newark, NJ 07102, USA \\ ${ }^{2}$ Instituto de Astrofisica de Canarias, Via Lactea s/n, La Laguna, E38200 Tenerife, Spain \\ Correspondence should be addressed to E. Pallé, epalle@iac.es
}

Received 16 June 2009; Accepted 9 November 2009

Academic Editor: Taro Kotani

Copyright ( $) 2010$ P. R. Goode et al. This is an open access article distributed under the Creative Commons Attribution License, which permits unrestricted use, distribution, and reproduction in any medium, provided the original work is properly cited.

The overall reflectance of sunlight from Earth is a fundamental parameter for climate studies. We have designed and implemented small aperture, remote control telescopes in Big Bear Solar Observatory in California and in Tenerife in the Canary Islands. These telescopes observe the earthshine to obtain a global mean terrestrial reflectance utilizing a coronagraph-like design for long exposures of the dark of the Moon and have internal moving parts in the optical train, which presented some design and control problems.

\section{Introduction}

For a decade, we have been measuring the Earth's reflectance by observing the earthshine, which is sunlight reflected from the Earth to the Moon and retroflected to the nighttime Earth. These observations provide an absolutely calibrated measure of the terrestrial albedo-a fundamental climate parameter.

For our observations, we have utilized a terrestrial determination of the Earth's global albedo from an old, and largely forgotten method. That is, an absolutely calibrated, large-scale albedo can be determined by measuring the amount of sunlight reflected from the Earth and in turn, back to the Earth from the dark portion of the face of the Moon (earthshine) and taking the ratio of that to the brightside (moonshine) signal; see Figure $1[1,2]$. In Figure 1, the fiducial points used in the measurements are shown in a single image in which the brightside is heavily filtered. The most important historical program of earthshine measurements was carried out by Danjon [3, 4] from a number of sites in France. Danjon estimated his uncertainty at roughly 5\% (ignoring his appreciable systematic error from an incorrect determination of the Moon's reflectivity). Our measurements are about an order of magnitude more precise than his estimates, in large part because we have better measurement technologies. We have also eliminated his systematic error by correctly measuring the scattering from the Moon as a function of the phase of the Moon [1]. At about 1\% precision on individual nights, our terrestrial estimates of the Earth's albedo have a precision comparable to that from satellites like ERBE with around the same value [5], and to those of the CERES instrumentation, of around $1 \%$ [6] making our method sufficiently precise to usefully complement satellite measurements.

The earthshine observations reveal a large decadal variability in the Earth's reflectance [7], which is yet not fully understood, but which is in line with other satellite and ground-based global radiation data (see $[8,9]$ and references therein). In order to continue to contribute to a better understanding of the Earth's radiation budget continuous observations from a global network of robotic telescopes are necessary.

The modern earthshine observations began in Big Bear Solar Observatory (BBSO) using a small-aperture telescope requiring an operator who controlled the swapping between observations of the brightside of the Moon (moonshine) with very short exposures, and the dark side (earthshine) with very long exposures. Changing to a highly automated, remotely operated telescope was more difficult than one might imagine because of the internal moving parts in the optical train operating during the near continuous switching between moonshine and earthshine observations, and the continuous variation of the lunar phase. 


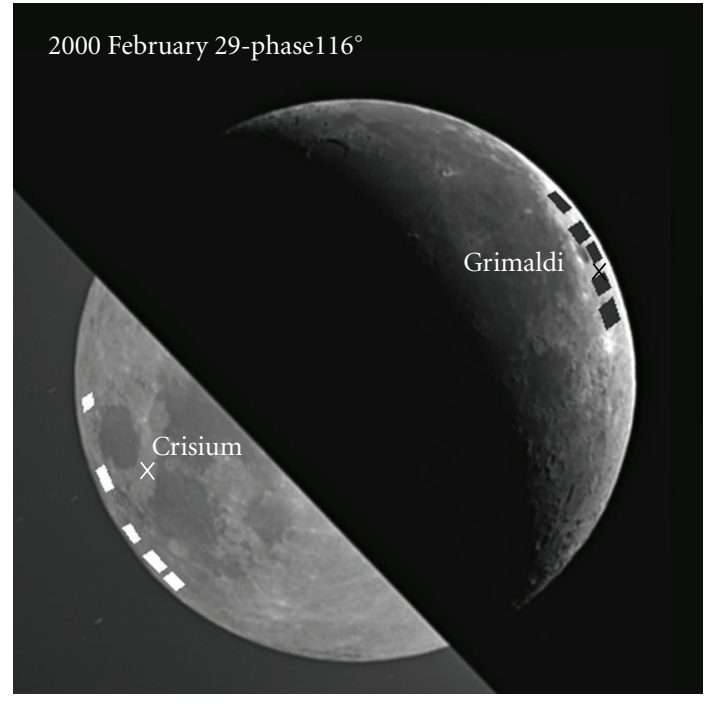

Figure 1: The moon showing the brightside and the earthshine. The Grimaldi side is in the moonshine (brightside) and the Crisium side is in the earthshine. Our ten highlands fiducial patches used in the observations made from BBSO are indicated. The crosses give the approximate positions of Danjon's $[3,4]$ fiducial patches. Goode et al. [11] used one fiducial patch on each side, and on the Crisium side it is the one closest to the white cross, while on the Grimaldi side, it is the one immediately above the black cross. In the image, the lunar phase is $115^{\circ} .9$, near a declining quarter moon. Unlike the moonshine, the earthshine is flat across the disk. The flatness is due to the uniform, incoherent back-scattering (non-Lambertian), in contrast to the forward scattering of sunlight occurring in the sunlit lunar crescent surface.

The telescope needs to function like a coronagraph because of stray light problems associated with long exposures to collect earthshine images. We now have built, tested, calibrated, and deployed two automatic telescopes for observing the earthshine. The first is in Big Bear Solar Observatory at $2 \mathrm{~km}$ above sea level in Southern California, while the second is in the Observatorio del Teide in the Canary Islands; see Figure 2. Two sites widely spaced in longitude are necessary to obtain global coverage. For instance, the BBSO telescope cannot see much of the North America or longitudes near the Europe-Asia boundary. A third site is working in Crimea Observatory, in Ukraine, but it is not a robotic, but a manually operated telescope. However that site has already allowed us a first comparison of data from two separate locations [10]. If funding is available we plan to increase our network to a maximum of $6-8$ telescopes.

\section{Why Earthshine Photometry Matters for Climate}

The net power deposited in the terrestrial atmosphere and surface depends on the solar irradiance and the Earth's shortwavelength (0.15-4.9 microns) albedo

$$
P_{\text {in }}=C \pi R_{e}^{2}(1-A),
$$

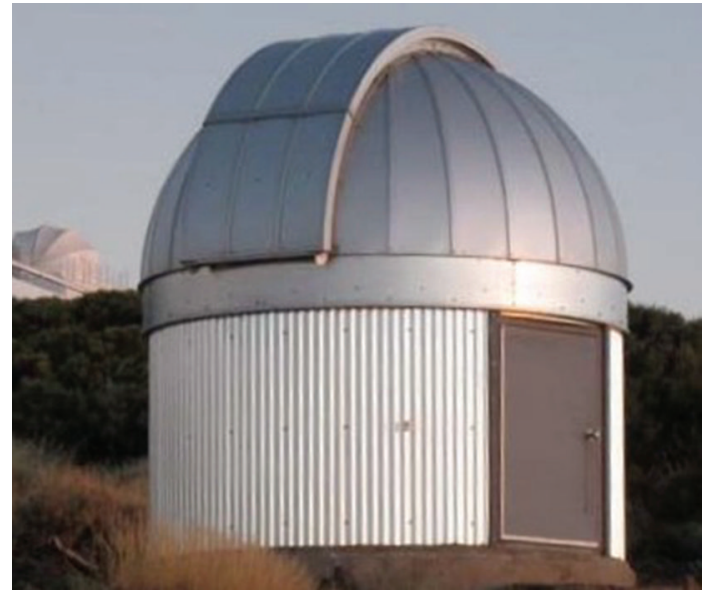

Figure 2: Earthshine dome in the Canaries. Part of the German Gregor dome may be seen in the background.

where $C$ is the solar constant (adjusted for the Sun-Earth distance), $R_{e}$ is the Earth's radius, and $A$ is the short-wavelength Bond albedo (the amount of sunlight reflected back to space by the atmosphere and surface of the Earth). Subsequently, the short-wavelength, incoming power is reradiated back into space at thermal or long-wavelengths (peaks near $\sim 10$ 15 microns), where

$$
P_{\text {out }}=4 \pi R_{e}^{2} \sigma T_{\text {toa }}^{4}
$$

and where $\sigma$ is the Stefan-Boltzmann constant and $T_{\text {toa }}$ $(\sim 255 \mathrm{~K})$ is the effective temperature of the Earth (defined with unit emissivity). $T_{\text {toa }}$ is a physically averaged longwave emission temperature at about $5.5 \mathrm{~km}$ height in the atmosphere (this "top of the atmosphere" or "toa" temperature depends on wavelength and cloud cover; altitudes from 0 to $30 \mathrm{~km}$ contribute to this emission). One can relate that temperature to a more relevant global climate parameter like the globally averaged surface temperature $T_{\text {sur }}$ by introducing a greenhouse forcing parameter $G\left[\mathrm{~W} / \mathrm{m}^{2}\right]$, which is defined as the difference between the emission at the top of the atmosphere and the surface. The forcing $G$ increases with an increasing concentration of greenhouse gasses. After Raval and Ramanathan [12], one can define the normalized greenhouse effect $g$ as $g=G / \sigma T_{\text {sur. }}^{4}$. Then the outgoing power can be written as

$$
P_{\text {out }}=4 \pi R_{e}^{2} \sigma(1-g) T_{\text {sur }}^{4}
$$

If the planet is in radiative equilibrium, $P_{\text {in }}=P_{\text {out }}$, then we have

$$
T_{\text {sur }}^{4}=\frac{C}{4 \sigma(1-g)}(1-A)
$$

This means that the Bond albedo, together with solar irradiance and the greenhouse effect, directly controls the Earth's temperature. Global warming would result if either $A$ decreased or $g$ or $C$ increased. 


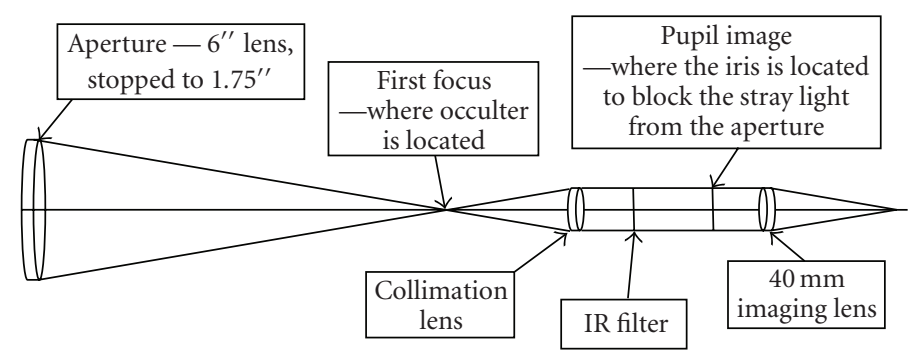

Figure 3: Schematic of the earthshine telescope optical train. At prime focus during the earthshine exposures, the moonshine blocker translates and rotates to its proper position to shield out the brightside. At the image of the pupil, the edges of the entrance aperture are blocked to reduce stray light. The earthshine camera chip is at the second focus.

\section{Observational Challenges to Automate an Earthshine Telescope}

A schematic of the the robotic telescopes is shown in Figure 3. Building the first robotic telescope required some care because it needs to translate/rotate in and out a blocking filter that blocks the scattered light from the sunlit moon during exposures on the earthshine side of the moon. The blocking filter is located at the first telescope focus. The second telescope focus is at the camera. In between, the telescope has a Lyot stop at the pupil image to block the stray light introduced by the edge of the entrance aperture. The telescope needs this "anticoronagraphic" structure to reduce stray light during very long exposures $(\sim 1$ minute of the earthshine, as opposed to the $\sim 10 \mathrm{~ms}$ exposures of the brightside). The source of the stray light in the dark side observations is light from the brightside of the Moon scattered by the atmosphere. The telescope was designed and built with stray light contamination in mind. In fact, we tested bright lights inside the dome and they only had a measurable effect if they were directed straight into the telescope along the path of the moonlight. A picture of the box housing the earthshine hardware in the Canaries is shown in Figure 4.

The dome, telescope, and coronagraph-like features are all under remote control. One can remotely open the dome, take flat fields, point the telescope, and then observations go on their own along the night, but can also be closed down from afar if conditions call for it.

\section{The Earthshine Telescope}

4.1. Controlling the Earthshine Telescope. The Earthshine robotic telescope and its dome have several hardware parts that are under software-control. These are the following.

(1) The camera: we use a Princeton Instruments PhotonMax 512 camera $(512 \times 512$ pixels, $8.2 \times 8.2 \mathrm{~mm}$ CCD size, 16 bit, high efficiency camera).

(2) The shutter: we use a $6 \mathrm{~mm}$ Vincent Associates UniBlitz LS6 shutter. This shutter can operate in the range from $2 \mathrm{~ms}$ up.

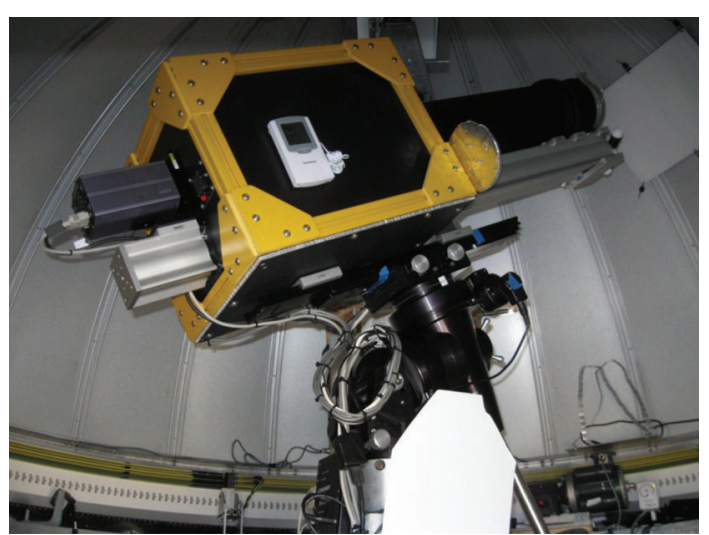

FIgure 4: The earthshine telescope shown inside its dome. The light-shielding yellow box extends about one-half meter along the optical axis.

(3) The occulter stages: we installed two Standas motorized stages, one is rotational with $50 \mathrm{~mm}$ aperture and another is translation stage. The occulter is mounted on the rotation stage and the rotation stage is in turn mounted on the translation stage.

(4) The dust cap at the end of the telescopes tube.

(5) The dome: the dome consists of three controllable parts: azimuth motor (dome rotation), the lower shutter door, and the upper shutter. To read the position of the dome we use a barcode tape (with barcodes evenly spaced approximately every inch) mounted on moveable part of the dome and a barcode scanner.

(6) The telescope mount: the telescope is installed on a Losmandy Titan equatorial mount.

To control various hardware parts, we use three Galil Motion Control's controllers. Two of them are 4-axis DMC2132 and one is an IO board IOC-7007. One of the DMC2132's controls the occulter stages and another one controls the cap. The IOC-7007 is used to control the dome's shutter, both the door and the upper shutter. We also use a relay board installed inside of the earthshine computer. The relay board controls the dome's azimuth motor. The computer's serial ports are used to set and trigger exposure via a UniBlitz 
shutter, to set various options on the camera and to control the mount. We also use two remotely controlled (via web interface) power switches to turn on/off power on various pieces of hardware, including the computer. If the main control computer hangs, we can reboot it remotely with the power switches. We installed several web cameras to watch and see that everything is working nominally.

The earthshine telescope software controls consist of two programs: ESCameraControl, which controls everything on the telescope with the exception of the dust cap and DomeControl, which controls the dome and the dust cap.

4.2. Remote Observing Sequence. The earthshine telescope is a remotely controlled telescope rather than a fully robotic telescope. This means that an observer (whose physical presence is not required) needs to send the commands to setup and start the observations. There are several steps in the remote observing sequence.

(1) Open the dome with the help of the DomeControl program, the remote "observer" initializes the azimuth control (read current position of the dome) and starts the dome's automatic movement. The dome will position the shutter opening according to the positions of the Moon and the telescope thus the moonlight shines on the camera.

(2) Remotely start the ESCameraControl program and open the dust cap.

(3) Observer points the telescope at the Moon (the program calculates position of the Moon) and put camera in the "live image" mode making sure that the Moon is in the field of view.

(4) With the help of the occulter control window, using linear and rotational movements of the stages find two positions of the occulter, occulter "in" and occulter "out." In the "out" position, the occulter is completely out of the field of view. Then set "in" position such that the occulter blocks illuminated side of the Moon (moonshine) and leaves earthshine side visible (like in Figure 1).

(5) After setting the "in" and "out" positions (which do not differ much from night to night) start-so-called "cycle". During one cycle of observations, the software first moves the occulter "out" and takes 10 moonshine images, then moves the occulter "in" and takes 1, longer, earthshine image. The program repeats this cycle until stopped by the operator. The program automatically adjusts exposure time so the image counts stay in the same range. After 30 images the program also takes dark frames for both exposures (moonshine and earthshine).

(6) At the end of observations the operator stops the cycle, parks the mount, and rotates the dome to some predefined position so the telescope is pointed toward a white screen mounted on the surface of the dome. Then the operator turns on the flat field lights mounted on the end of the telescope and takes flat field images.

(7) After the observations are completed for the evening, the observer closes the dome shutter and turns off the hardware, including the camera. All of the above steps are done remotely by the observer, using a remote desktop control software called UltraVNC. We installed the UltraVNC server on the earthshine computer and the UltraVNC client on the operator's controlling computer.

Steps (1)-(5) take about 15 minutes at the beginning of the observations each night. After which, the remote observer may largely ignore his/her control computer. The computer has an alarm system that sounds and shows red on the screen, if a problem arises. The observations will stop automatically if the warnings are ignored. Steps (6)-(7) take about 10 minutes at the end of observations each night. This remote observing sequence is ideal for the Canary Islands where observers remotely control several synoptic type telescope each night from a remote mountaintop control room a few hundred meters from the earthshine dome.

\section{Conclusions}

Future observations of the earthshine from a planned global network of robotic telescopes will provide an even more valuable tool, complementary to satellite data, for the study of changes in the short-wave forcing of the Earth's climate. We plan to expand our two station remote-control network to a global one with several stations.

\section{Acknowledgments}

P. R. Goode and S. Shoumko gratefully acknowledge the support from NASA-NNX08BA22G. Research by E. Pallé was supported by a "Ramon y Cajal" fellowship.

\section{References}

[1] J. Qiu, P. R. Goode, E. Pallé, et al., "Earthshine and the Earth's albedo: 1. Earthshine observations and measurements of the lunar phase function for accurate measurements of the Earth's Bond albedo," Journal of Geophysical Research D, vol. 108, no. D22, article 4709, pp. 1-22, 2003.

[2] E. Pallé, P. R. Goode, V. Yurchyshyn, et al., "Earthshine and the Earth's albedo: 2. Observations and simulations over 3 years," Journal of Geophysical Research D, vol. 108, no. D22, article 4710, pp. 1-17, 2003.

[3] A. Danjon, "Recherches sur la photométrie de la lumière cendrée et l'albedo de la terre," Annales de l'Observatoire de Strasbourg, vol. 2, pp. 165-180, 1928.

[4] A. Danjon, "Albedo, color, and polarization of the Earth," in The Earth as a Planet, G. P. Kuiper, Ed., pp. 726-738, University of Chicago Press, Chicago, Ill, USA, 1954.

[5] E. F. Harrison, P. Minnis, B. R. Barkstrom, V. Ramanathan, R. D. Cess, and G. G. Gibson, "Seasonal variation of cloud radiative forcing derived from the Earth Radiation Budget Experiment," Journal of Geophysical Research, vol. 95, no. D11, pp. 18687-18703, 1990.

[6] S. Kato, N. G. Loeb, and C. K. Rutledge, "Estimate of top-of-atmosphere albedo for a molecular atmosphere over ocean using Clouds and the Earth's Radiant Energy System measurements," Journal of Geophysical Research D, vol. 107, no. 19, p. 4396, 2002.

[7] E. Pallé, P. R. Goode, P. Montañés-Rodríguez, and S. E. Koonin, "Changes in Earth's reflectance over the past two decades," Science, vol. 304, no. 5675, pp. 1299-1301, 2004.

[8] E. Pallé, P. Montañés-Rodríguez, P. R. Goode, S. E. Koonin, M. Wild, and S. Casadio, "A multi-data comparison of shortwave 
climate forcing changes," Geophysical Research Letters, vol. 32, no. 21, Article ID L21702, 4 pages, 2005.

[9] E. Pallé, P. R. Goode, and P. Montañés-Rodríguez, "Interannual variations in Earth's reflectance 1999-2007," Journal of Geophysical Research, vol. 114, Article ID D00D03, 2009.

[10] E. Pallé, P. R. Goode, P. Montañés-Rodríguez, S. E. Koonin, and V. Rumyantsev, "Toward a global earthshine network: first results from two stations," Geophysical Research Letters, vol. 32, no. 11, Article ID L11803, 4 pages, 2005.

[11] P. R. Goode, J. Qiu, V. Yurchyshyn, et al., "Earthshine observations of the Earth's reflectance," Geophysical Research Letters, vol. 28, no. 9, pp. 1671-1674, 2001.

[12] A. Raval and V. Ramanathan, "Observational determination of the greenhouse effect," Nature, vol. 342, no. 6251, pp. 758-761, 1989. 

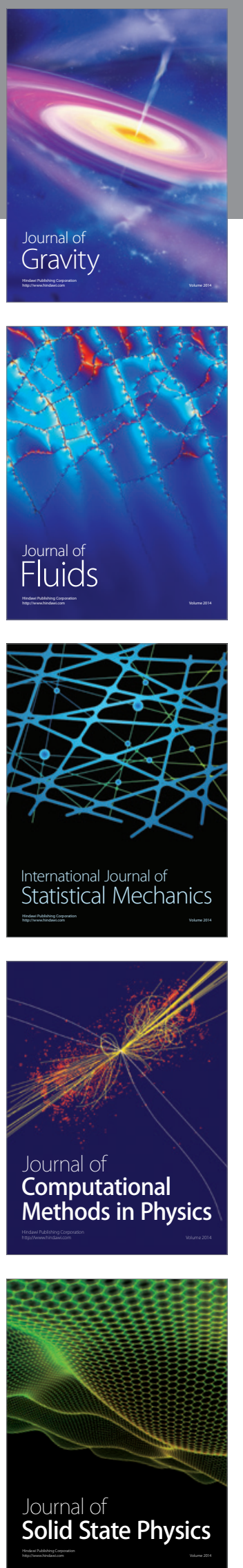

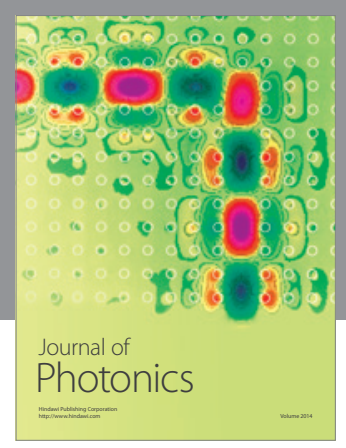

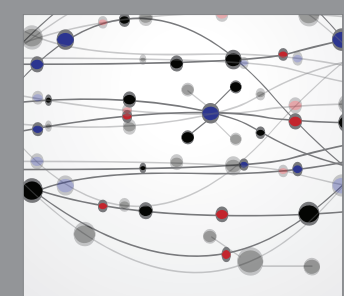

The Scientific World Journal
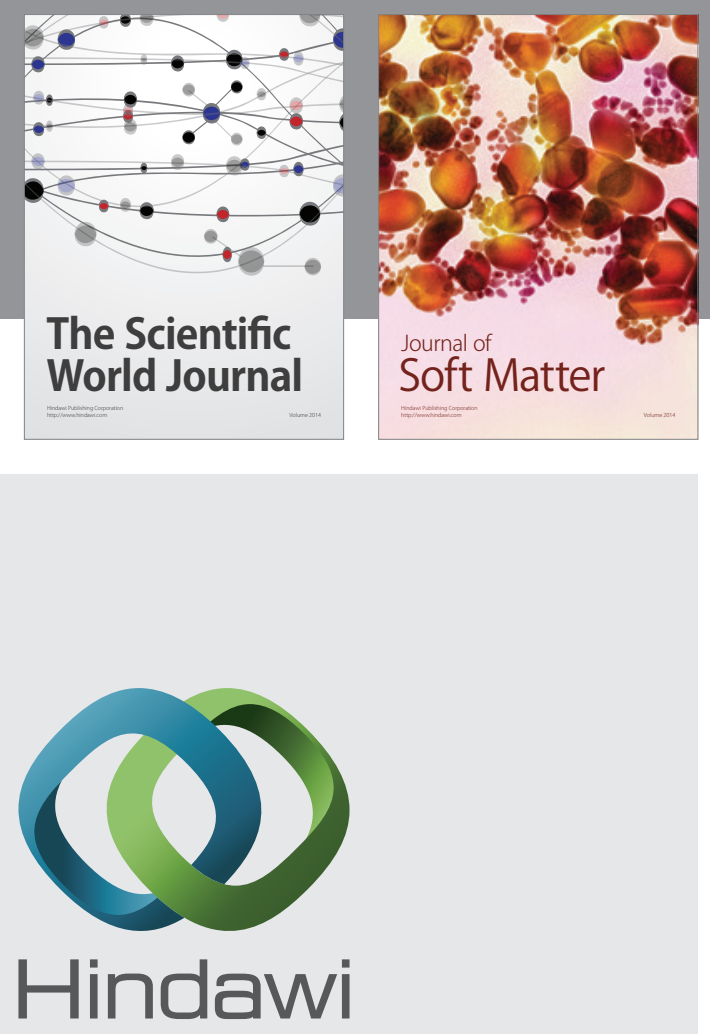

Submit your manuscripts at

http://www.hindawi.com
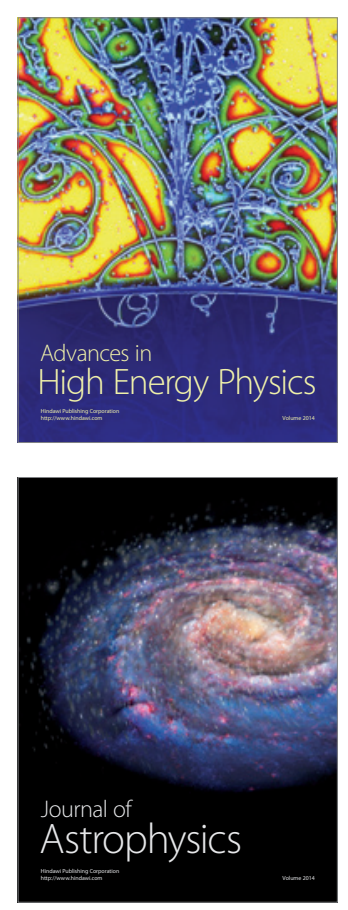
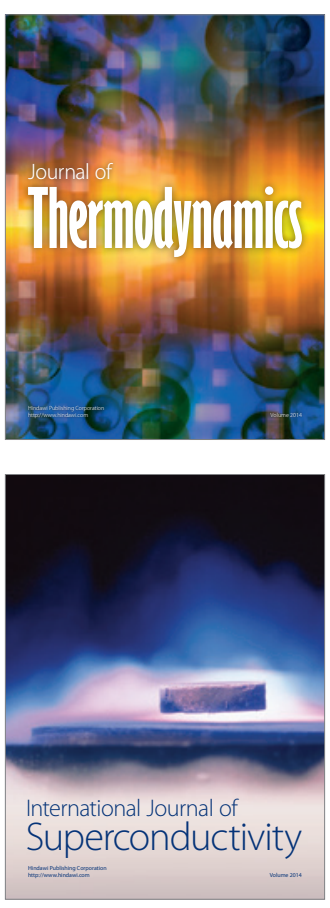
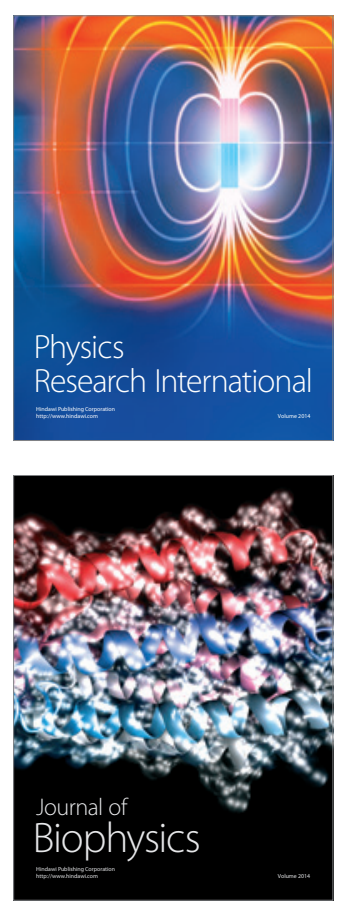
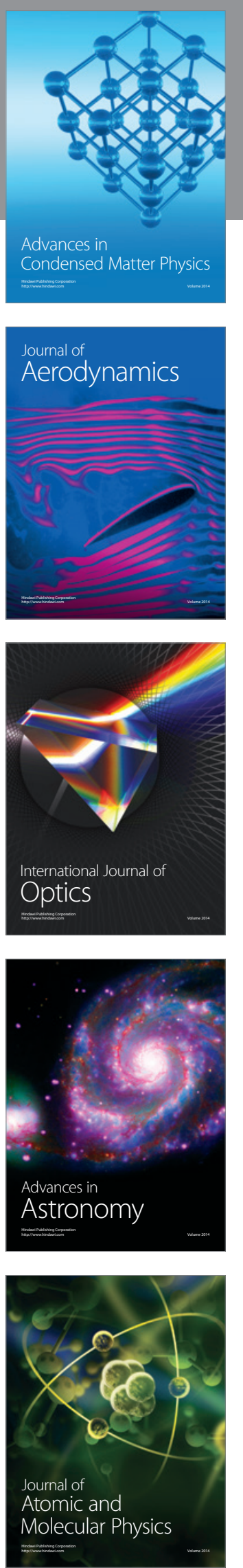